\title{
Effect of Gold Sodium Thiomalate and Its Thiomalate Component on the In Vitro Expression of Endothelial Cell Adhesion Molecules
}

\author{
Peter M. Newman, ${ }^{\star}$ Shing Shun Tony To,* Bruce G. Robinson, ${ }^{\ddagger}$ Valentine J. Hyland, ${ }^{\star}$ and Leslie Schrieber ${ }^{\star}$ \\ * Sydney University Department of Rheumatology and ${ }^{\ddagger}$ Molecular Genetics Unit, Royal North Shore Hospital, Sydney, 2065, Australia
}

\begin{abstract}
Endothelial adhesion molecules play an important role in the tissue recruitment of leukocytes in inflammatory conditions such as rheumatoid arthritis. We have investigated the effect of the antirheumatic drug gold sodium thiomalate on adhesion molecule protein and mRNA expression in cultured human endothelial cells. Gold sodium thiomalate inhibited cytokine (TNF, IL-1, IL-4)-stimulated expression of vascular cell adhesion molecule-1 and E-selectin but not intercellular adhesion molecule-1 on endothelial cells. Gold sodium thiomalate also suppressed TNF-stimulated increases in vascular cell adhesion molecule-1 and E-selectin mRNA levels but had no effect on intercellular adhesion molecule-1 mRNA. Thiomalate (mercaptosuccinate), but not gold thioglucose or D-penicillamine, mimics the effect of gold sodium thiomalate at equimolar concentrations. We propose that the inhibition of vascular cell adhesion molecule-1 and E-selectin expression by gold sodium thiomalate is due to its thiomalate and not its gold component. Gold sodium thiomalate has a direct effect on endothelial adhesion molecule expression, and this may contribute to its antiinflammatory activity. (J. Clin. Invest. 1994. 94:1864-1871.) Key words: cell adhesion molecules, drug effects • endothelium - intercellular adhesion molecule-1 - vascular cell adhesion molecule-1 • E-selectin
\end{abstract}

\section{Introduction}

Leukocyte infiltration is a key component of chronic inflammation (1). Adhesion molecules on endothelial cells play an important role in targeting and facilitating leukocyte migration out of the blood vessels, in response to an inflammatory stimulus.

Stimulation of endothelial cells in vitro with inflammatory cytokines such as TNF results in increased expression of inter-

\footnotetext{
Address correspondence to Peter M. Newman, Department of Rheumatology, Royal North Shore Hospital, St. Leonards, NSW, 2065, Australia.

Received for publication 10 January 1994 and in revised form 7 July 1994.
}

1. Abbreviations used in this paper: $\mathrm{BSO}$, DL-buthionine- $[S, \mathrm{R}]$-sulfoximine; D-pen, D-penicillamine; GSTM, gold sodium thiomalate; GTG, gold thioglucose; HUVE, human umbilical vein endothelium; ICAM1, intercellular adhesion molecule-1; MTT, 3,-4,5 dimethylthiazol-2,5 diphenyl tetrazolium bromide; TM, thiomalate; VCAM-1, vascular cell adhesion molecule-1.

J. Clin. Invest.

(C) The American Society for Clinical Investigation, Inc. 0021-9738/94/11/1864/08 $\$ 2.00$

Volume 94, November 1994, 1864-1871 cellular adhesion molecule-1 (ICAM-1), ${ }^{1}$ vascular cell adhesion molecule-1 (VCAM-1), and E-selectin (2, 3). ICAM-1 is expressed on a wide variety of cell types but only at low levels on unstimulated endothelial cells (4). TNF or IL-1 stimulation induces increased expression of ICAM-1 that reaches a plateau after $24 \mathrm{~h}$ and remains elevated for at least $2 \mathrm{~d}$. VCAM-1 is not constitutively expressed and peaks after 10-24 h of TNF stimulation. E-selectin expression is restricted to endothelial cells (4). While there is no basal expression of E-selectin on endothelial cells, continuous TNF or IL-1 stimulation results in a maximum level after $6 \mathrm{~h}$ which then declines $(2,4)$. The transient expression of E-selectin in vitro may not always be relevant in vivo. Sustained in vivo E-selectin expression has been observed in chronically inflamed tissues including psoriatic skin and rheumatoid synovium $(5,6)$. Moreover, in vitro and in vivo IFN- $\gamma$ prolongs E-selectin expression. It has been suggested that IFN- $\gamma$, released at sites of inflammation, may extend the duration of E-selectin expression $(7,8)$.

Elevated levels of TNF have been demonstrated in rheumatoid arthritic joints $(9,10)$. Increased expression of VCAM-1 (11), E-selectin (6), CD31 (PECAM-1), and $\beta_{3}$ integrin subunit (vitronectin receptor) (12) is present on the endothelial cells of rheumatoid synovium compared with normal or osteoarthritic synovium. A number of antiinflammatory drugs have been shown to affect adhesion molecules and/or leukocyte-endothelial interactions (for review see reference 13). Notably, the expression of ICAM-1 on endothelial cells is reduced by colchicine (14) and dexamethasone (15). Furthermore, synovial biopsies from rheumatoid patients treated with gold sodium thiomalate (GSTM) show decreased E-selectin expression (16).

Despite the extensive body of literature on the mechanism by which gold compounds alleviate rheumatoid arthritis (17, 18), no dominant paradigm has emerged. GSTM inhibits monocyte oxygen radical generation (19) and $\mathrm{Fc}$ and $\mathrm{C} 3$ receptor expression and chemotaxis (20). Moreover, GSTM has effects on endothelial cells including inhibition of cell proliferation (21) and HLA-DR expression (22).

Studies in mice and humans indicate that GSTM readily dissociates in vivo. The gold component rapidly binds to sulfhydryl groups on proteins while free thiomalate (TM) is either rapidly excreted or taken up by tissues. In mice, $6 \mathrm{~h}$ after injection, only $0.1 \%$ of the thiomalate remains circulating, while $\sim 50 \%$ is incorporated into tissues (23). These observations, and the lack of correlation between blood gold concentrations and therapeutic effect, have led to the proposal that TM is responsible for some or all of the antiinflammatory effect of $\operatorname{GSTM}(24,25)$. In this study, we have investigated the in vitro effect of GSTM and TM on TNF-stimulated expression of ICAM-1, VCAM-1, and E-selectin on endothelial cells.

\section{Methods}

Reagents. Bovine lung heparin, BSA, di-sodium EDTA, Tween 20, 2,2'azino-bis-(3-ethylbenzthiazoline-6-sulfonic acid), and 3,-4,5 dimethyl- 
thiazol-2,5 diphenyl tetrazolium bromide (MTT) were all supplied by Sigma Chemical Co. (St Louis, MO). Gelatine and phenol were purchased from BDH Chemicals Ltd. (Poole, United Kingdom). Chloroform and Triton X-100 were from BDH (Kilsyth, Victoria, Australia). Collagenase was obtained from Worthington Biochemical Corp. (Freehold, NJ). Medium 199 with Hanks' salts was supplied by ICN Biomedicals, Inc. (Costa Mesa, CA). Recombinant TNF was a generous gift from Dr. Debbie Rathjen (Peptide Technology, Sydney, Australia). Recombinant IL- $1 \alpha$ and IL-4 were supplied by Boehringer Mannheim GmbH (Mannheim, Germany). $75-\mathrm{cm}^{2}$ plastic cell culture flasks were purchased from Corning Glass Works (Corning, NY). 60-cm-diameter culture dishes (LUX brand) were obtained from Nunc, Inc. (Naperville, IL). 96-well plates were manufactured by Nunc (Kamstrup, Denmark) or by Biofusion (New South Wales, Australia). FCS was bought from Cytosystems (New South Wales, Australia). Glutaraldehyde and formaldehyde were supplied by Ajax Chemicals (New South Wales, Australia). Monoclonal antibodies to VCAM-1 and E-selectin were purchased from British Biotechnology (Abingdon, United Kingdom) and Becton Dickinson Immunocytometry Systems (San Jose, CA), respectively. AntiICAM-1 was a generous gift from Dr. Andrew Boyd (Walter and Eliza Hall Institute, Melbourne, Australia). The Vectastain kit was obtained from Vector Labs, Inc. (Burlingame, CA), and the peroxidase-conjugated goat anti-mouse was purchased from Tago, Inc. (Burlingame, CA). Sheep anti-mouse $F\left(a^{\prime}\right)_{2}$ conjugated to FITC was from Silenus (Hawthorn, Victoria, Australia). ${ }^{51} \mathrm{Cr}$-labeled sodium chromate was supplied by Amersham International (Little Chalfont, Buckinghamshire, United Kingdom). Guanidine thiocyanate was manufactured by International Biotechnologies, Inc. (New Haven, CT). Agarose was bought from Promega Corp. (Madison, WI). GeneScreen nylon membrane was from New England Nuclear Research Products (Boston, MA). SDS was manufactured by ICN Biomedicals (Cleveland, $\mathrm{OH}$ ). Dextran sulfate was purchased from Pharmacia AB (Uppsala, Sweden). Sheared salmon sperm DNA was supplied by Calbiochem-Novabiochem (La Jolla, CA). All DNA probes were obtained as gifts. ICAM-1 (1.5 kb excised from pGEM7 with XbaI and HindIII) was from Dr. Andrew Boyd. VCAM1 (3 kb of clone VCAM1E11 excised from CDM8 with NotI) was from Biogen (Cambridge, MA). E-selectin (1,841 bp from XhoI digest of pCDM8) was from Dr. Eugene Butcher (Department of Pathology, Stanford University, Stanford, CA). Mouse actin ( 600 bp excised from Blue Scribe with PstI) was from Dr. Bruce Spiegelman (Dana Farber Cancer Institute, Boston, MA). Probes were labeled with $3,000 \mathrm{Ci} /$ mmol $\left[\alpha-{ }^{32} \mathrm{P}\right] \mathrm{dCTP}$ supplied by Amersham International using a random primer kit (Boehringer Mannheim GmbH).

Preparation of drugs. GSTM (May and Baker, Melbourne, Australia) was dissolved in water at $30 \mathrm{mM}$ (based on monomeric weight) and allowed to stand at room temperature until the yellow color disappeared $(\sim 1 \mathrm{~h})$. DL-buthionine-[S,R]-sulfoximine (BSO; Sigma Chemical Co.) was dissolved in water at $6 \mathrm{mM}$. Thiolactate, thioglycolate, gold thioglucose (GTG), thioglucose (all from Sigma Chemical Co.), TM (mercaptosuccinic acid; Aldrich Chemical Co., Milwaukee, WI), D-penicillamine (D-pen) (Eli Lilly, Sydney, Australia), and L-cysteine hydrochloride (Ajax, Sydney, Australia) were dissolved in water at $\mathbf{3 0}$ $\mathrm{mM}$ and adjusted to $\mathrm{pH} \mathrm{6-8}$ with $\mathrm{NaOH}$ if necessary. Buffering to physiological $\mathrm{pH}$ occurred upon mixing with basal medium and placement in the $5 \% \mathrm{CO}_{2}$ atmosphere of the cell culture incubator. These solutions were diluted at least 30 -fold in basal medium before incubating with cells. L-cystine has a low solubility at $\mathrm{pH} 7(26)$, so it could not be prepared as a concentrate in water. Instead, a solution was prepared by dissolving cystine $(0.072 \mathrm{mg} / \mathrm{ml}$; Hopkin and Williams, Essex, United Kingdom) in basal medium (10\% FCS in Medium 199) and adding $\mathrm{NaOH}$ to $10 \mathrm{mM}$. Upon dissolution, the medium was returned to neutral $\mathrm{pH}$ by the addition of $\mathrm{HCl}$. Basal medium contains $100 \mu \mathrm{M}$ cystine as an essential ingredient (ICN Biomedicals) so the concentration of this solution was $400 \mu \mathrm{M}$. A final concentration of $300 \mu \mathrm{M}$ cystine was realized when $400 \mu \mathrm{M}$ cystine was diluted 2:1 with basal medium containing TNF. The effect of $300 \mu \mathrm{M}$ cystine was compared with that of basal medium which had undergone the same rise and fall in $\mathrm{pH}$.

Human umbilical vein endothelial (HUVE) cell culture. HUVE cells were isolated as previously described (27). Briefly, human umbilical veins were filled with collagenase solution, incubated at $37^{\circ} \mathrm{C}$ for 10 min, flushed with Medium 199, and cells were collected by centrifugation. Cells were grown in gelatin $(0.2 \%)$ coated $75-\mathrm{cm}^{2}$ flasks and maintained in growth medium consisting of Medium 199 supplemented with $20 \%$ FCS, $50 \mu \mathrm{g} / \mathrm{ml}$ bovine lung heparin, and 1:100 dilution of bovine brain extract (28). HUVE cells were used up to passage five.

Cell ELISA. Endothelial cells were plated at confluent density into the wells of gelatin-coated 96-well plates. After overnight incubation, the growth medium was removed and replaced with basal medium (10\% FCS in Medium 199) containing TNF (10 ng/ml) and test drugs or with basal medium alone. Cells were incubated for $24 \mathrm{~h}$ (unless otherwise stated), then washed in PBS with $0.05 \%$ Tween 20 (PBS/Tween), fixed in $0.025 \%$ glutaraldehyde for $10 \mathrm{~min}$, washed and stored for $1-3 \mathrm{~d}$ at $4^{\circ} \mathrm{C}$ in PBS/Tween containing $0.02 \%$ gelatin.

Adhesion molecules were detected using ELISA by incubating cells with a primary antibody to either ICAM-1 (1:3,000 ascites), VCAM-1 $(0.1 \mu \mathrm{g} / \mathrm{ml})$, or E-selectin $(0.1 \mu \mathrm{g} / \mathrm{ml})$. In the case of the ICAM-1 assay, cells were incubated with a peroxidase-conjugated goat anti-mouse secondary antibody. VCAM-1 and E-selectin antibodies were detected with the Vectastain ABC kit by incubating the cells with a biotinylated secondary antibody followed by avidin-conjugated peroxidase. Finally, the expression of ICAM-1, VCAM-1, and E-selectin was quantitated by the addition of the peroxidase substrate $2,2^{\prime}$-azino-bis-(3-ethylbenzthiazoline-6-sulfonic acid). The absorbance of each well was measured at $414 \mathrm{~nm}$, and the background at $492 \mathrm{~nm}$ was subtracted. Within each experiment, wells containing endothelial cells were treated in quadruplicate with each dose of drug. At each dose, mean \pm SD was calculated.

Flow cytometry. HUVE cells were plated at confluent density onto 60-cm-diameter cell culture dishes. After overnight incubation, the growth medium was removed and replaced with basal medium with and without TNF and GSTM. After $24 \mathrm{~h}$ of incubation, the plates were washed with PBS, and the cells were detached with $5 \mathrm{mM}$ EDTA. $10^{5}$ cells were incubated on ice with mAbs to ICAM-1 (1:4,000 ascites) and VCAM-1 $(1 \mu \mathrm{g} / \mathrm{ml})$ for $30 \mathrm{~min}$. Cells were washed twice with PBS containing $0.25 \%$ BSA and then treated on ice for half an hour with anti-mouse-FITC (1:50 dilution) secondary antibody. After two more washes cells were analyzed by flow cytometry (Coulter Corp., Hialeah, FL).

${ }^{5 l} \mathrm{Cr}$ release assay. HUVE cells were plated at confluent density into the wells of gelatin-coated 96-well plates. After overnight incubation, cells were labeled with ${ }^{51} \mathrm{Cr}$ by incubating with basal medium containing sodium chromate $(320 \mathrm{kBq} / \mathrm{ml})$ for $2 \mathrm{~h}$. Each well was washed three times and then incubated with TNF \pm GSTM. After $24 \mathrm{~h}$ of incubation, the supernatant was sampled and counted in a $\gamma$-counter (LKB-Wallac, Turku, Finland). Maximal release (max counts) of ${ }^{51} \mathrm{Cr}$ was assessed by the addition of $1 \%$ Triton X-100 detergent, while incubating with basal medium alone recorded minimum release (min counts). All treatments were performed in triplicate, and the percentage of ${ }^{51} \mathrm{Cr}$ released was calculated from the means of the triplicates as follows:

Percent release

$$
=100 \times(\text { test counts }-\min \text { counts }) /(\max \text { counts }-\min \text { counts }) .
$$

MTT cell viability assay. HUVE cells were incubated for $24 \mathrm{~h}$ with TNF and the highest concentration of each test drug as per cell ELISA. Cells were then washed with PBS and incubated $2-3 \mathrm{~h}$ with MTT $(0.4$ $\mathrm{mg} / \mathrm{ml}$ ) in basal medium (29). Cells were washed again with PBS, and formazan crystals were dissolved with $100 \mu \mathrm{l}$ DMSO. The absorbance of each well was measured at $540 \mathrm{~nm}$. The effect of each drug on cell viability was calculated from the means of quadruplicates as follows:

Percent viability $=100 \times($ Test absorbance/Drug-free absorbance $)$.

Northern blot analysis. Endothelial cells were grown to confluence in $75-\mathrm{cm}^{2}$ flasks and treated in triplicate with TNF and either GSTM or TM at 0,30 , or $1,000 \mu \mathrm{M}$ in basal medium. One flask was treated with basal medium alone (no TNF). After $24 \mathrm{~h}$ of incubation, cells 


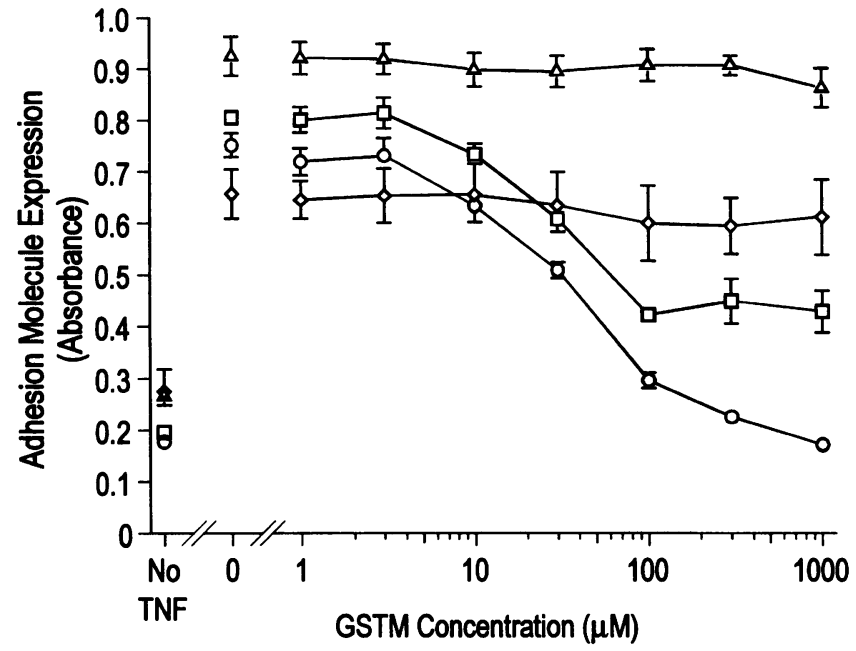

Figure 1. Adhesion molecule expression on HUVE cells incubated with TNF and GSTM. VCAM-1 expression after $24 \mathrm{~h}(0)$, E-selectin expression after $24 \mathrm{~h}(\square)$, E-selectin after $6 \mathrm{~h}(\triangle)$, and ICAM-1 expression after $24 \mathrm{~h}(\diamond)$ were measured using a cell ELISA. Mean \pm SD was calculated from four replicate wells. Where SD is not shown, it is less than or equal to the size of the markers. The slope of the linear regression line, based on three independent experiments, was statistically significant for VCAM-1 and E-selectin after $24 \mathrm{~h}(P<0.0001)$ but not for ICAM-1 or E-selectin after $6 \mathrm{~h}$.

were removed using trypsin/EDTA, and total RNA was isolated using a guanidine thiocyanate-phenol-chloroform method based on that of Chomczynski and Sacchi (30).

$15 \mu \mathrm{g}$ of denatured total RNA was electrophoresed through a $1.4 \%$ agarose gel containing $1.25 \mathrm{M}$ formaldehyde and then was transferred to GeneScreen nylon membrane and ultraviolet cross-linked (Stratalinker; Stratagene, La Jolla, CA). The replicate treatments were processed independently throughout the RNA isolation and electrophoresis.

DNA probes of VCAM-1, mouse $\beta$-actin, E-selectin, and ICAM1 were labeled with $\left[\alpha-{ }^{32} \mathrm{P}\right] \mathrm{dCTP}$ by the random primer method and incorporated at least $10^{9} \mathrm{cpm} / \mu \mathrm{g}$. The membrane was prehybridized overnight in $5 \times$ sodium chloride sodium phosphate EDTA buffer (SSPE) (31), 2\% SDS, $10 \%$ dextran sulfate, and $1 \mu \mathrm{g} / \mathrm{ml}$ sheared salmon sperm DNA at $65^{\circ} \mathrm{C}$. The membrane was hybridized overnight with VCAM-1 $\left(>10^{6} \mathrm{cpm} / \mathrm{ml}\right)$ in the prehybridization solution and was then washed at high stringency in $0.1 \times \mathrm{SSPE}, 0.1 \%$ SDS at $65^{\circ} \mathrm{C}$. Autoradiography and quantification of band intensities was performed using a Phosphor-Imager (Molecular Dynamics, Inc., Sunnyvale, CA). The same procedure was used to probe with actin, E-selectin, and then ICAM-1. To avoid overlapping bands, the membrane was stripped by boiling in $10 \mathrm{mM}$ Tris, $1 \mathrm{mM}$ EDTA, 1\% SDS for a half an hour before probing with E-selectin and ICAM-1. The relative transcript accumulation for a given band was calculated from the ratio of counts in the band to counts in the actin band of that same lane.

Statistical analysis. All experiments examining the effect of a drug on adhesion molecule expression were performed at least twice. Results were obtained using HUVE cells derived from different individuals and gave very similar results. Therefore, one representative experiment is shown in the figures. However, data from replicate experiments were pooled before undertaking statistical analysis. Adhesion molecule expression was standardized using the formula:

Standardized expression

$=($ Test Abs - Min Abs)/(Max Abs - Min Abs);

where Test Abs is mean absorbance (of quadruplicate wells) at test concentration of drug, Min Abs is mean absorbance (of quadruplicate

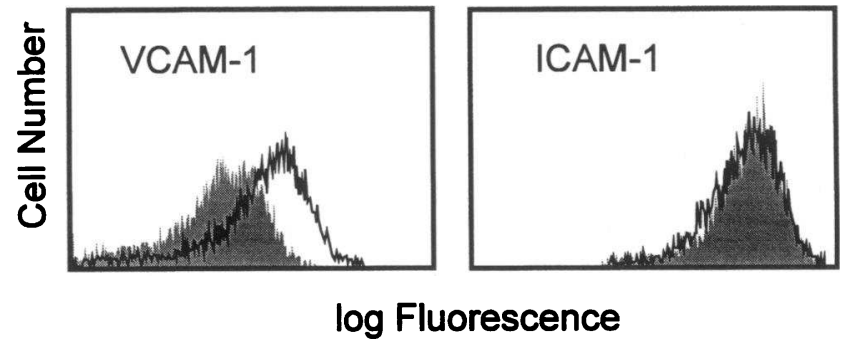

Figure 2. Flow cytometry profile of HUVE cells incubated for $24 \mathrm{~h}$ with TNF (black trace) or TNF $+1,000 \mu \mathrm{M}$ GSTM (gray area). Indirect immunofluorescence was used to identify cells labeled with monoclonal antibodies to either VCAM-1 or ICAM-1.

wells) without TNF, and Max Abs is mean absorbance (quadruplicate wells) when drug concentration is zero.

Standardized measures of adhesion molecule expression were plotted against the $\log _{10}$ of the drug concentration, and a linear regression line was fitted using least squares (32). A $t$ test was used to determine if the slope of this regression line was significantly (i.e., $P<0.05$ ) different from zero (32). A significant slope is evidence that the drug exerts a dose-dependent effect.

\section{Results}

The effect of GSTM on the cytokine-stimulated expression of endothelial adhesion molecules was investigated using a cell ELISA (Fig. 1). Incubation of endothelial cells with TNF for $24 \mathrm{~h}$ increased the expression of ICAM-1, VCAM-1, and Eselectin. However, when GSTM was also added, VCAM-1 and E-selectin expression was suppressed in a concentration-dependent manner (Fig. 1). By contrast, the expression of ICAM-1 remained unaffected. TNF stimulation for $6 \mathrm{~h}$ also elevated Eselectin expression but no dose-dependent effect of GSTM was observed over this period (Fig. 1).

The cell ELISA method used to detect E-selectin and VCAM-1 differed slightly from that used for ICAM-1. To exclude this as the explanation for the difference between the effect of GSTM on VCAM-1 compared with ICAM-1, another assay system was evaluated. Flow cytometry was used to compare the effect of GSTM on VCAM-1 and ICAM-1 expression. Fig. 2 illustrates that $1,000 \mu \mathrm{M}$ GSTM decreases VCAM-1 expression but not ICAM-1 expression, a finding consistent with the cell ELISA result.

To ascertain whether the effect of GSTM was specific for TNF-activated cells, we investigated the GSTM response of HUVE cells stimulated with IL-1 or IL-4. When IL-1 was used (in place of TNF) to stimulate adhesion molecule expression, GSTM again exhibited a selective dose-dependent inhibition of VCAM-1 and E-selectin, but not ICAM-1, expression after 24 h (Fig. 3). Similarly, GSTM inhibited the IL-4-stimulated expression of VCAM-1. IL-4 did not elevate ICAM-1 or E-selectin expression.

To determine which component(s) of GSTM was responsible for the inhibition of VCAM-1 and E-selectin expression, we examined the effect of GTG and TM. Like GSTM, GTG is an antirheumatic gold(I) compound, but the thiol ligand binding the gold is based on glucose rather than malic acid. D-pen was also examined because it is used clinically and like TM has a reduced thiol group. Fig. 4 illustrates that thiomalate exhibited 

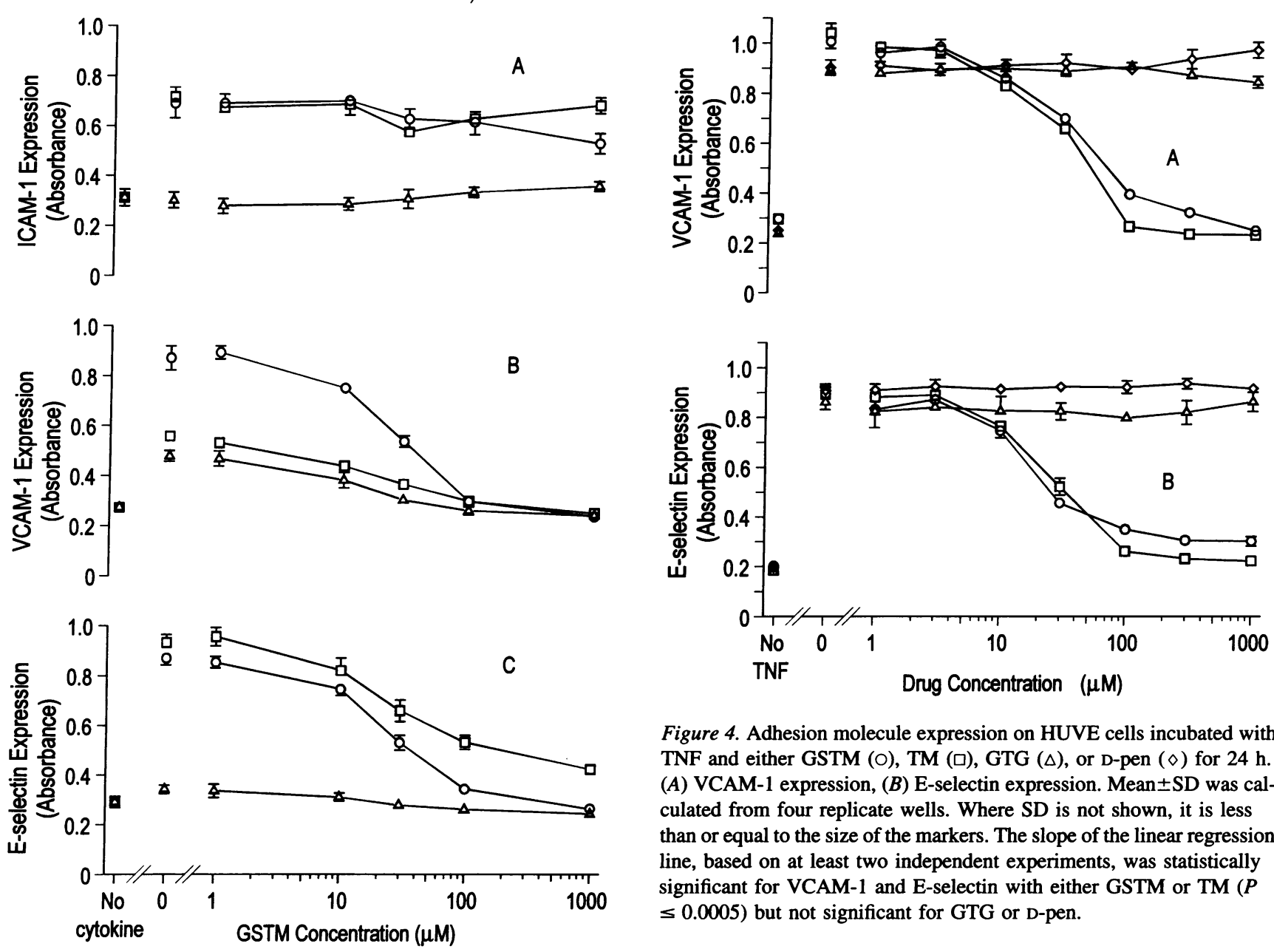

Figure 4. Adhesion molecule expression on HUVE cells incubated with TNF and either GSTM $(O)$, TM ( $\square)$, GTG $(\Delta)$, or D-pen $(\diamond)$ for $24 \mathrm{~h}$. $(A)$ VCAM-1 expression, $(B)$ E-selectin expression. Mean \pm SD was calculated from four replicate wells. Where SD is not shown, it is less than or equal to the size of the markers. The slope of the linear regression line, based on at least two independent experiments, was statistically significant for VCAM-1 and E-selectin with either GSTM or TM ( $P$ $\leq 0.0005$ ) but not significant for GTG or D-pen.

Figure 3. Adhesion molecule expression on HUVE cells incubated with GSTM and either TNF (O), IL-1 ( $\square$ ), or IL-4 $(\triangle)$ for $24 \mathrm{~h}$. (A) ICAM1 expression, $(B)$ VCAM-1 expression, $(C)$ E-selectin expression. Mean $\pm S D$ was calculated from four replicate wells. Where SD is not shown, it is less than or equal to the size of the markers. The slope of the linear regression line, based on two independent experiments, was statistically significant when VCAM-1 expression was stimulated by TNF $(P<0.005)$, IL-1 $(P<0.05)$, or IL-4 $(P<0.005)$. GSTM also had a significant effect on E-selectin, but not ICAM-1, expression during TNF $(P<0.0005)$ or IL-1 $(P<0.0005)$ stimulation. IL-4 did not stimulate either ICAM-1 or E-selectin expression.

a similar effect to GSTM while D-pen and GTG showed no effect over the range of $1-1,000 \mu \mathrm{M}$.

A range of other thiol compounds was studied in an attempt to elucidate the specificity and mechanism of GSTM and TM inhibition of VCAM-1 and E-selectin expression. Thiolactate and thioglycolate were chosen as they are structurally related to TM. The possible importance of a reduced thiol group was further investigated using cysteine and cystine. Cysteine has a free SH group, while cystine is composed of two cysteine molecules joined by a disulfide bond. Thioglucose, which is related to GTG, was also evaluated.

Thioglycolate and cysteine had a modest inhibitory effect at high concentrations, while thioglucose had no significant effect on VCAM-1 or E-selectin expression (Fig. 5). Thiolactate had a slight effect on VCAM-1 but not E-selectin expression.

None of these thiol compounds approached the potency of TM in the inhibition of VCAM-1 and E-selectin expression.

The concentration range over which cystine could be studied was limited. Cystine $(100 \mu \mathrm{M})$ is an ingredient of the basal medium (ICN Biomedicals), and the solubility of cystine (26) limited the maximum useable concentration to $300 \mu \mathrm{M}$. HUVE cells incubated for $24 \mathrm{~h}$ with TNF and $300 \mu \mathrm{M}$ cystine displayed significant $(P<0.0001, t$ test, $n=4)$ reductions in VCAM-1 (less 14\%) and E-selectin (less 16\%) expression relative to the basal concentration of $100 \mu \mathrm{M}$ cystine.

The effect of BSO (an inhibitor of glutathione synthesis) was studied to test the hypothesis that glutathione was involved in the inhibition of VCAM-1 and E-selectin expression by GSTM and TM. Over the concentration range of 0.2-200 $\mu \mathrm{M}$, the slope of the linear regression line, based on three independent experiments, was not significantly different from zero. In a typical experiment, the absorbance due to VCAM-1 expression ranged between 1.11 and 1.12 , while the absorbance due to $\mathrm{E}$ selectin ranged from 0.82 to 0.84 .

Kinetic studies were undertaken to further evaluate the similarities in the effects of GSTM and TM on endothelial cell adhesion molecule expression. Fig. 6 illustrates the time course for the inhibition of VCAM-1 and E-selectin expression by 30 $\mu \mathrm{M}$ GSTM or TM. In the absence of TNF and GSTM or TM, the background expression of the adhesion molecules remained low. In the presence of TNF alone, VCAM-1 expression reached a plateau at $12 \mathrm{~h}$ and remained elevated at $24 \mathrm{~h}$. By contrast, 

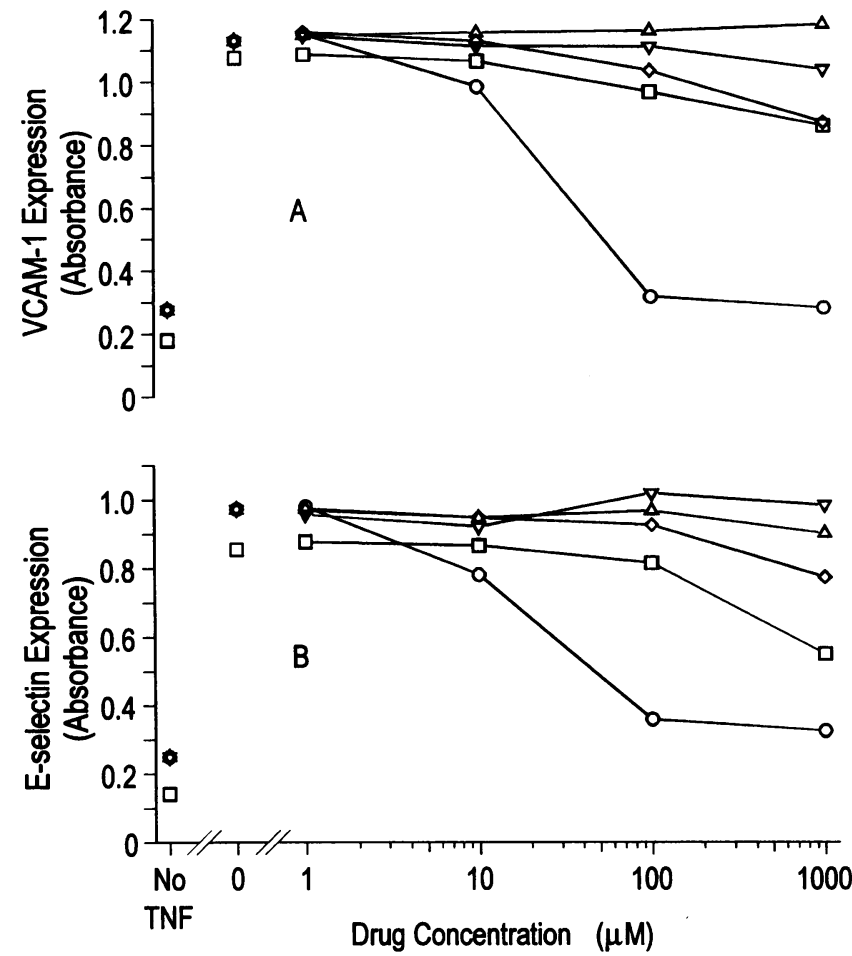

Figure 5. VCAM-1 $(A)$ and E-selectin $(B)$ expression on HUVE cells incubated for $24 \mathrm{~h}$ with TNF and either thiolactate $(\nabla)$, thioglycolate $(\diamond)$, TM (O), cysteine $(\square)$, or thioglucose $(\Delta)$. Mean was calculated from four replicate wells. SD is not shown, to maintain clarity. The slope of the regression line, based on at least three independent experiments, was significant for TM $(P \leq 0.0005)$ and thioglycolate $(P<0.005)$ but not for thioglucose in both the VCAM-1 and E-selectin assays. Thiolactate had a statistically significant effect on VCAM-1 $(P<0.005)$ but not on E-selectin expression. Cysteine had a more significant effect on E-selectin $(P<0.005)$ than VCAM-1 $(P<0.05)$ expression.

E-selectin expression peaked between 6 and $12 \mathrm{~h}$ and then declined. When cells were incubated with TNF and either GSTM or TM, the expression of both VCAM-1 and E-selectin decreased compared with cells treated with TNF alone. This effect was noted at $6 \mathrm{~h}$ and became more marked by $12 \mathrm{~h}$.

A ${ }^{51} \mathrm{Cr}$ release assay was used to investigate whether GSTM or TM had cytotoxic effects on endothelial cells that could explain the reduction in adhesion molecule expression. There was no significant difference $\left(P=0.105\right.$, ANOVA) in ${ }^{51} \mathrm{Cr}$ release between cells incubated with or without GSTM or TM (Fig. 7). An MTT cytotoxicity assay, which relies on the activity of mitochondrial dehydrogenases (29), recorded a $20 \%$ drop in cell viability at $1,000 \mu \mathrm{M}$ GSTM or TM. $1,000 \mu \mathrm{M}$ cysteine and $300 \mu \mathrm{M}$ cystine both decreased viability by $10 \%$ relative to $0 \mu \mathrm{M}$ concentrations. None of the other drugs investigated displayed any toxicity in the MTT assay.

Having established that GSTM and TM inhibited the expression of VCAM-1 and E-selectin protein, we investigated their effect on adhesion molecule mRNA levels. Northern blot analysis showed that endothelial cells stimulated with TNF had higher levels of ICAM-1, VCAM-1, and E-selectin mRNA than cells incubated without TNF (Fig. 8). The presence of $1,000 \mu \mathrm{M}$ GSTM or TM reduced the (TNF-stimulated) levels of VCAM-1 and E-selectin mRNA to near basal levels but had no effect on ICAM-1 transcript. Band intensities were used to quantitate
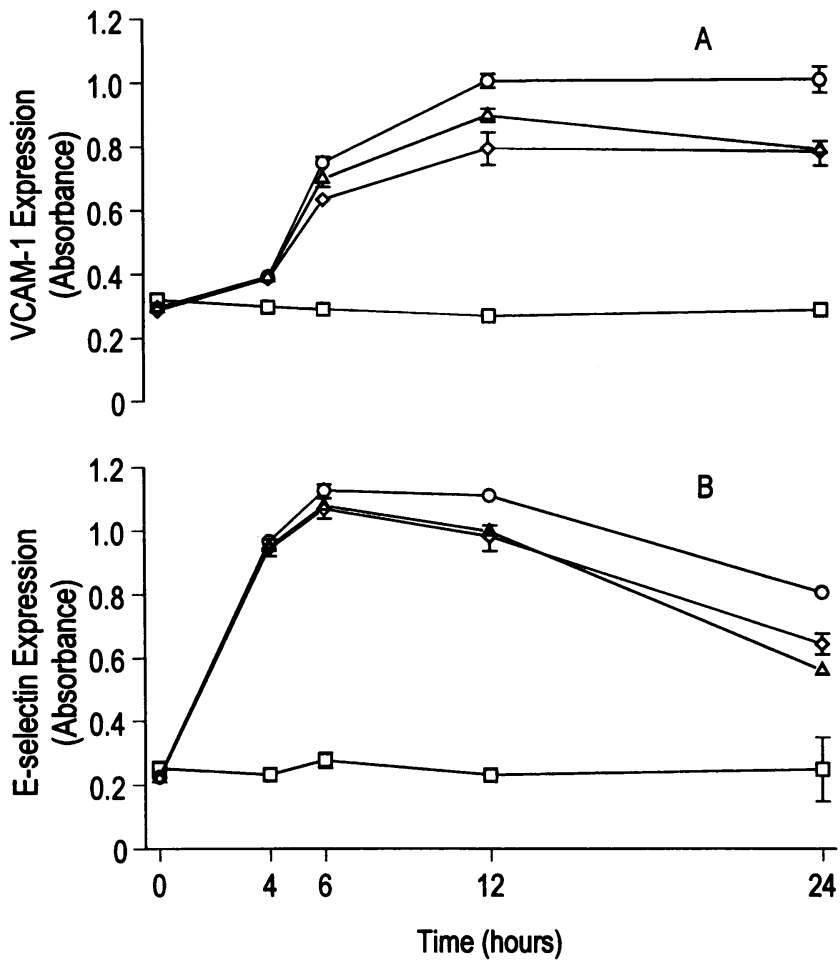

Figure 6. Kinetics of expression of VCAM-1 $(A)$ and E-selectin $(B)$ on HUVE cells incubated with TNF (O), TNF $+30 \mu \mathrm{M}$ GSTM $(\triangle)$, TNF $+30 \mu \mathrm{M}$ TM $(\diamond)$, or basal medium alone ( $\square)$. Mean \pm SD was calculated from three replicate wells. Where SD is not shown, it is less than or equal to the size of the markers.

mRNA species relative to the actin band (Fig. 9). Fisher's significant difference test (33) established that only at $1,000 \mu \mathrm{M}$ GSTM or TM was VCAM-1 mRNA significantly $(P<0.005)$ reduced relative to the "TNF only" control. $30 \mu \mathrm{M}$ GSTM or TM had no significant effect $(P>0.05)$ on VCAM-1 mRNA. In contrast, E-selectin transcript was significantly $(P<0.005)$ reduced by $30 \mu \mathrm{M}$ concentrations. Incubation with $1,000 \mu \mathrm{M}$ GSTM or TM resulted in a further significant $(P<0.005)$ reduction in E-selectin mRNA levels. ICAM-1 transcript accu-

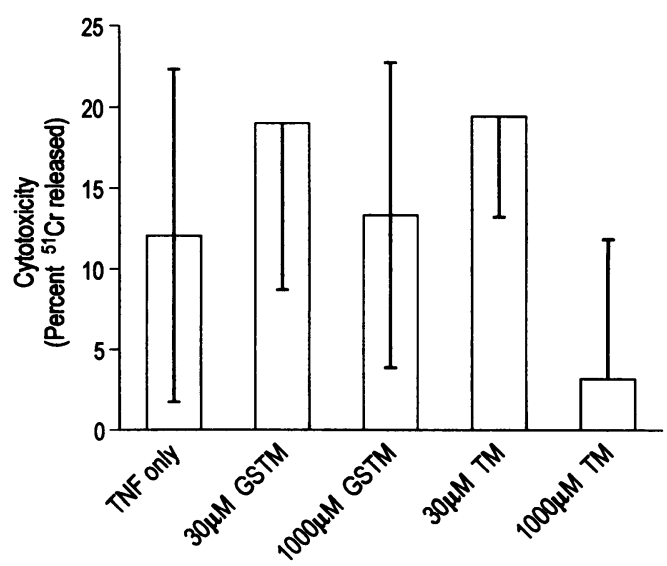

Figure 7. ${ }^{51} \mathrm{Cr}$ release assay of HUVE cells incubated for $24 \mathrm{~h}$ with TNF and 0,30 , and 1,000 $\mu \mathrm{M}$ concentrations of either GSTM or TM. Mean \pm SD was calculated from four replicate wells. 


\section{$\begin{array}{llllllllllllllll}1 & 2 & 3 & 4 & 5 & 6 & 7 & 8 & 9 & 10 & 11 & 12 & 13 & 14 & 15 & 16\end{array}$

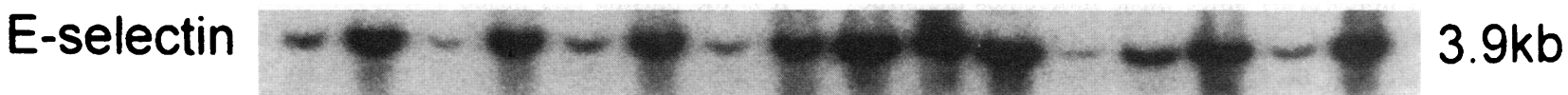
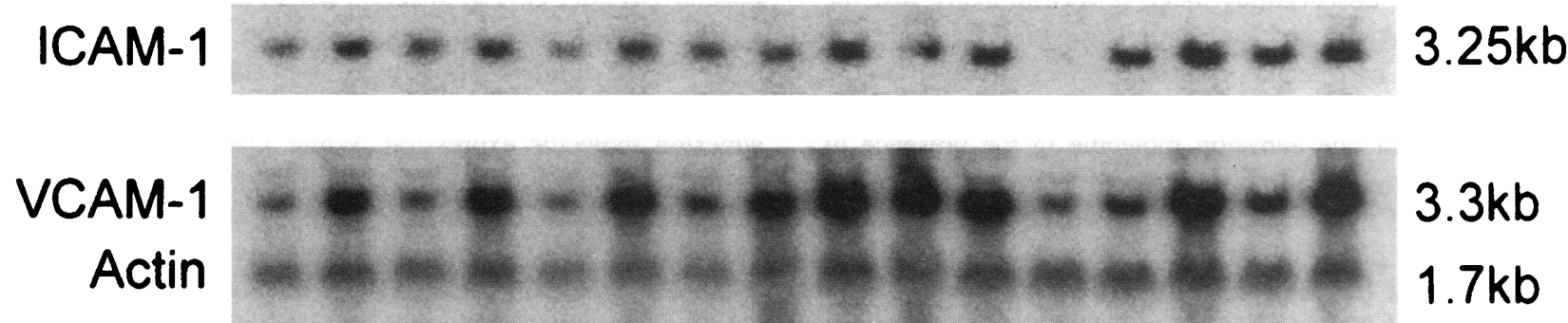

Figure 8. Northern blot analysis of HUVE cells incubated for $24 \mathrm{~h}$ with TNF and either GSTM or TM. The membrane was sequentially probed with VCAM-1, actin, E-selectin, and ICAM-1. Lanes 1, 5, and 13, TNF + 1,000 $\mu \mathrm{M}$ TM; lanes 2, 6, and 14, TNF + 30 $\mu \mathrm{M}$ TM; lanes 3, 7, and 15 , TNF + 1,000 $\mu \mathrm{M}$ GSTM; lanes 4, 8, and 16, TNF + $30 \mu \mathrm{M}$ GSTM; lanes 9-11, TNF only; and lane 12, basal medium alone.

mulation was not significantly affected by any of the concentrations of GSTM and TM tested. The effects of GSTM and TM on mRNA levels can be summarized: for VCAM-1 the effect of $1,000 \mu \mathrm{M}>30 \mu \mathrm{M} \approx$ TNF only; for E-selectin $1,000 \mu \mathrm{M}$ $>30 \mu \mathrm{M}>$ TNF only; for ICAM-1 $1,000 \mu \mathrm{M} \approx 30 \mu \mathrm{M}$ $\approx$ TNF only.

\section{Discussion}

Many mechanisms have been proposed for the antirheumatic action of GSTM. Recent in vivo studies have suggested that GSTM may act by reducing endothelial adhesion molecule expression. Corkill et al. (16) found decreased E-selectin (ELAM-
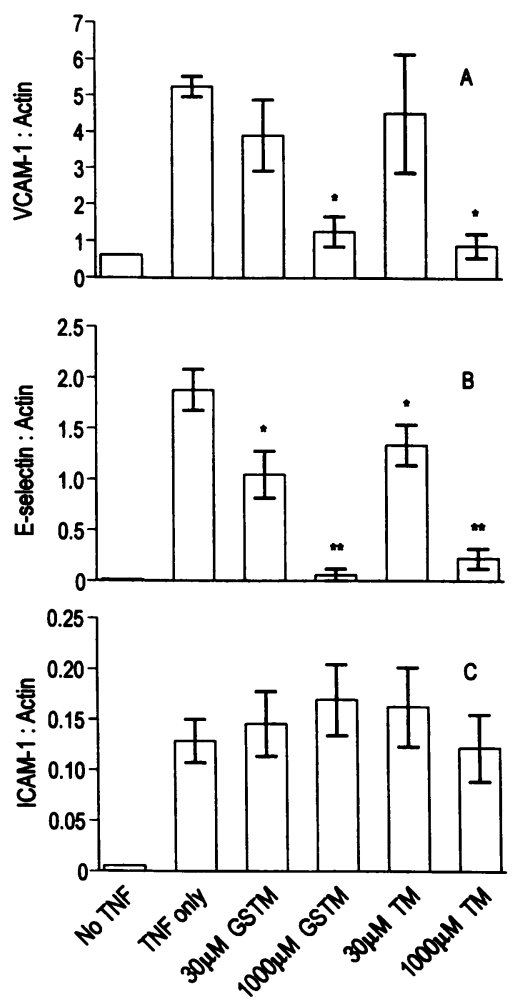

Figure 9. Analysis of band intensities on the Northern blot (Fig. 8). Relative transcript accumulation is expressed as the ratio of counts in the adhesion molecule band to counts in the actin band. Means \pm SD were calculated from triplicates, except in the case of No TNF where the single sample was omitted from any statistical analysis. Graphs show relative transcript accumulation for VCAM-1 $(A)$, Eselectin $(B)$, ICAM-1 (C). Fisher's least significant difference test was used to compare means within each graph. *Means are significantly different $(P<0.005)$ from the TNF only control. **Means are significantly below $(P$ $<0.005) 30 \mu \mathrm{M}$ GSTMor TM-treated cells.
1) expression on the synovial endothelium of rheumatoid arthritis patients treated with GSTM. However, it is unclear whether this is a direct effect of GSTM on synovial endothelium or an indirect effect by reducing inflammation and cytokine levels. We used an in vitro model to investigate the effect of GSTM on endothelial adhesion molecule expression and found that GSTM exhibited a selective and dose-dependent inhibition of VCAM-1 and E-selectin expression (Fig. 1). From this we conclude that GSTM can act directly on endothelial cells to inhibit VCAM-1 and E-selectin expression.

Cronstein et al. (15) observed that the inhibition of ICAM1 and E-selectin by dexamethasone occurred with $\mathrm{IL}-1-$ and endotoxin-, but not TNF-, stimulated endothelial cells. We found that GSTM inhibited both the TNF- and IL-1-stimulated expression of VCAM-1 and E-selectin (Fig. 3). GSTM was also effective when IL-4 was used to selectively induce VCAM-1. This suggests that GSTM acts at a step common to the pathways by which these cytokines induce VCAM-1 and E-selectin expression. The effectiveness of GSTM, regardless of cytokine, is consistent with the hypothesis that the inhibition of adhesion molecule expression is a mechanism by which GSTM exerts some of its antiinflammatory properties in vivo.

GSTM dissociates into protein-bound gold and free thiomalate in vivo $(23,24)$. It is likely that a similar reaction occurs in vitro. The effect of GSTM on VCAM-1 and E-selectin expression could be due to the gold component, the thiomalate, or both. We demonstrated that TM, without gold, is sufficient to inhibit VCAM-1 and E-selectin expression (Fig. 4). As GTG showed no effect, we conclude that gold(I) is not required for the inhibition of adhesion molecule expression.

The thiol group is the chemical group that distinguishes GSTM and TM from most other compounds in the cell. To examine the importance of this group in the inhibitory activity of GSTM and TM on adhesion molecule expression, a number of other thiol compounds were tested. Neither D-pen, cysteine, cystine, thioglucose, thiolactate, nor thioglycolate at equimolar concentrations approached the degree of inhibition of VCAM1 and E-selectin expression that is produced by TM (Fig. 5). Thus, the mere presence of a thiol moiety per se is unlikely to explain the effect of GSTM and TM.

The observation that thiolactate $\left(\mathrm{CH}_{3} \mathrm{CH}(\mathrm{SH}) \mathrm{COO}^{-}\right)$and 
thioglycolate $\left(\mathrm{HSCH}_{2} \mathrm{COO}^{-}\right)$had little effect on adhesion molecule expression is noteworthy since these molecules are structurally related to $\mathrm{TM}\left({ }^{-} \mathrm{OOCCH}(\mathrm{SH}) \mathrm{CH}_{2} \mathrm{COO}^{-}\right.$). Thus, relatively small differences in chemical structure may result in marked biological differences.

A number of observations make it very unlikely that the inhibition of VCAM-1 and E-selectin expression by GSTM or TM is due to a nonspecific cytotoxic effect. Firstly, GSTM does not inhibit ICAM-1 expression. Thus, the cells are able to synthesize at least one adhesion molecule in the presence of GSTM. Secondly, TNF-stimulated endothelial cell viability, assessed using a ${ }^{51} \mathrm{Cr}$ release assay, was unaltered by GSTM or TM up to $1,000 \mu \mathrm{M}$ (Fig. 7). An MTT assay was used as a third independent indicator of cell viability. A small drop in cell viability at $1,000 \mu \mathrm{M}$ GSTM and TM was noted but is unlikely to account for the marked effect on VCAM-1 and Eselectin expression.

The inhibitory effect of GSTM and TM was further characterized by kinetic studies (Fig. 6). The time course of TNFstimulated VCAM-1 expression is consistent with previous reports $(2,4)$. The effect of GSTM and TM is apparent after 12 $h$ incubation, although there is a small difference in VCAM-1 expression after just $6 \mathrm{~h}$ between cells treated with TNF only and those with GSTM. Continuous TNF stimulation induced Eselectin expression to increase rapidly and peak between 6 and $12 \mathrm{~h}$. E-selectin expression decreased to $60 \%$ of the peak level by $24 \mathrm{~h}$. The prolonged E-selectin expression observed in our experimental system, compared with others (2), remains unexplained. After $6 \mathrm{~h}$ of incubation there is negligible difference between cells treated with and without GSTM despite E-selectin expression being maximal (Fig. 6). It is only after 12 and $24 \mathrm{~h}$ that the difference is evident. This suggests that there is a delay between the addition of GSTM or TM to the cells and the time when these drugs exert their effect. The entry of TM into cells is not rapid. Aguilera et al. (34) found that the intracellular concentration of thiomalate was only $15 \%$ of the $30 \mathrm{mM}$ concentration in the medium after $1 \mathrm{~h}$ of incubation. When we pretreated endothelial cells by incubating for $24 \mathrm{~h}$ with GSTM or TM alone, and then washed the cells and incubated them with TNF for $6 \mathrm{~h}$, a dose-dependent inhibition of E-selectin by GSTM and TM was observed (data not shown). Thus, by allowing TM and GSTM time to penetrate the cell and interact with its target molecule(s), we were able to demonstrate that these drugs inhibit E-selectin after $6 \mathrm{~h}$ of TNF stimulation.

Quantitative analysis of RNA isolated from HUVE cells showed that GSTM and TM inhibited VCAM-1 and E-selectin mRNA accumulation relative to actin mRNA (Fig. 9). ICAM1 mRNA was unaffected. Thus GSTM and TM selectively reduce both the protein and mRNA accumulation of E-selectin and VCAM-1. We propose that the reduction in protein expression of these molecules is due, at least in part, to the decrease in their mRNA levels. Whether GSTM and TM act by inhibiting transcription or by promoting transcript degradation remains to be determined.

Conventional GSTM therapy results in serum gold concentrations in the range of $15-35 \mu \mathrm{M}$ and is associated with a decrease in E-selectin expression on synovial endothelium. In our in vitro assay, $15 \mu \mathrm{M}$ GSTM decreased E-selectin and VCAM-1 expression by 20 and $30 \%$, respectively. Thus, it is plausible that GSTM exerts some of its antiinflammatory properties by decreasing VCAM-1 and E-selectin expression. However, as the decreased expression of adhesion molecules may be attributable to TM and not the gold component of GSTM, it is the plasma and tissue TM levels that are relevant for this discussion. Plasma TM peaks at $0.5 \mu \mathrm{M}$, a half hour after GSTM injection, and declines almost to zero by $2 \mathrm{~h}$ (24). From our in vitro data, it appears that the concentration and duration of plasma TM after GSTM injection would be insufficient to have an effect on adhesion molecule expression. However, intramuscular GSTM administration results in a substantial store of tissue-bound TM (25) that may be available to inhibit endothelial adhesion molecule expression. While TM is rapidly excreted during the first $6 \mathrm{~h}$ after GSTM administration, it then plateaus such that after $24 \mathrm{~h}$ only $60 \%$ of the injected dose has been voided (25). Thus, in rheumatoid arthritis, TM bound to cells within the synovium may be slowly released and may be available to reduce the expression of E-selectin and VCAM-1 on endothelial cells.

The observation that both GSTM and TM reduce endothelial adhesion molecule expression suggests that TM may be an effective antirheumatic compound. Previous workers have cited the structural similarities of TM to D-pen and the fact that GSTM dissociates into TM in vivo as supporting evidence (24). A preliminary trial of oral TM for the treatment of rheumatoid arthritis was very encouraging (35). However, these results have not been published as a complete paper. Furthermore, when the trial was independently repeated, oral TM was found to be ineffective and to cause adverse side effects (36). No trials involving parenteral administration of TM (analogous to GSTM therapy) have been reported. It may be that the route of delivery, and/or the association with gold, is critical for TM to decrease adhesion molecule expression in vivo.

TM is known to be a potent inhibitor of the enzyme glutathione peroxidase, as is D-pen, in a cell-free system (37). However, we observed that D-pen did not inhibit the expression of any of the adhesion molecules tested. Thus, assuming D-pen can penetrate the cell membrane to a similar degree as TM, it is unlikely that TM inhibits VCAM-1 expression by blocking glutathione peroxidase. In a further experiment, we evaluated the effect of BSO (an inhibitor of glutathione synthesis) on endothelial adhesion molecule expression. $200 \mu \mathrm{M}$ BSO eliminates glutathione, the substrate for glutathione peroxidase, after $24 \mathrm{~h}$ of incubation (38). Thus, by eliminating its substrate, the activity of glutathione peroxidase within the cells can be indirectly inhibited. However, we observed that $0.2-200 \mu \mathrm{M}$ BSO had no effect on VCAM-1 or E-selectin expression on TNF-stimulated endothelial cells. Thus, it is unlikely that the inhibition of VCAM-1 and E-selectin by TM is due to the inhibition of glutathione peroxidase.

A possible mechanism for the inhibition of adhesion molecule expression involves the second messenger cAMP. Elevated cAMP, induced by forskolin or isobutyl methylxanthine, inhibits VCAM-1 and E-selectin but not ICAM-1 expression on TNFstimulated endothelial cells (39). The similarity of this effect, to that which we observed with GSTM, suggests that GSTM may inhibit adhesion molecule expression by increasing cAMP in endothelial cells. In fact, Scheinberg et al. (20) observed increased cAMP in monocytes incubated in vitro with GSTM and suggested that this may be responsible for inhibited chemotaxis. However, in contrast, Lazarevic et al. (40) observed that GSTM inhibited the activity of adenylate cyclase from lymphocyte membranes. This would result in decreased intracellular cAMP. Thus, to resolve whether or not cAMP is involved in the inhibition of VCAM-1 and E-selectin by GSTM, it would 
be necessary to determine the effect of GSTM on endothelial cAMP and to correlate this with adhesion molecule expression.

In summary, we have demonstrated that GSTM inhibits the in vitro endothelial expression of VCAM-1 and E-selectin, but not ICAM-1. We propose that this inhibition is due to the thiomalate and not the gold component of GSTM and involves the reduction of mRNA for VCAM-1 and E-selectin. The decrease in endothelial adhesion molecule expression by GSTM may reduce the recruitment of leukocytes to rheumatoid synovium and thus may contribute to the antiinflammatory effects of this compound.

\section{Acknowledgments}

We are grateful to Dr. Malcolm King for performing flow cytometry and to Dr. Terry Longhurst for advice regarding the Northern blot. Dr. Malcolm Handel (Garvan Institute for Medical Research, St. Vincent's Hospital, Sydney, Australia) provided valuable discussion, and Mrs. Caro Badcock generously gave her time to discuss statistics.

This work was supported by the National Health and Medical Research Council of Australia and an equipment grant from the Rebecca L. Cooper Research Foundation.

\section{References}

1. Cotran, R. S., V. Kumar, and S. L. Robbins. 1989. Robbins Pathologic Basis of Disease. W. B. Saunders Co., Philadelphia. 1519 pp.

2. Wellicome, S. M., M. H. Thornhill, C. Pitzalis, D. S. Thomas, J. S. Lanchbury, G. S. Panayi, and D. O. Haskard. 1990. A monoclonal antibody that detects a novel antigen on endothelial cells that is induced by tumor necrosis factor, IL1, or lipopolysaccharide. J. Immunol. 144:2558-2565.

3. Veale, D., G. Yanni, S. Rogers, L. Barnes, B. Bresnihan, and O. Fitzgerald. 1993. Reduced synovial membrane macrophage numbers, ELAM-1 expression, and lining layer hyperplasia in psoriatic arthritis as compared with rheumatoid arthritis. Arthritis Rheum. 36:893-900.

4. Bevilacqua, M. P. 1993. Endothelial-leukocyte adhesion molecules. Annu. Rev. Immunol. 11:767-804.

5. Rohde, D., W. Schluter-Wigger, V. Mielke, P. von den Driesch, B. von Gaudecker, and W. Sterry. 1992. Infiltration of both T cells and neutrophils in the skin is accompanied by the expression of endothelial leukocyte adhesion molecule-1 (ELAM-1): an immunohistochemical and ultrastructural study. J. Invest. Dermatol. $98: 794-799$.

6. Koch, A. E., J. C. Burrows, G. K. Haines, T. M. Carlos, J. M. Harlan, and S. J. Leibovich. 1991. Immunolocalization of endothelial and leukocyte adhesion molecules in human rheumatoid and osteoarthritic synovial tissues. Lab. Invest. 64:313-320.

7. Leeuwenberg, J. F., E. J. von Asmuth, T. M. Jeunhomme, and W. A. Buurman. 1990. IFN-gamma regulates the expression of the adhesion molecule ELAM-1 and IL-6 production by human endothelial cells in vitro. J. Immunol. 145:2110-2114

8. Munro, J. M., J. S. Pober, and R. S. Cotran. 1989. Tumor necrosis factor and interferon-gamma induce distinct patterns of endothelial activation and associated leukocyte accumulation in skin of Papio anubis. Am. J. Pathol. 135:121-133.

9. Hopkins, S. J., and A. Meager. 1988. Cytokines in synovial fluid. II. The presence of tumour necrosis factor and interferon. Clin. Exp. Immunol. 73:8892.

10. Yocum, D. E., L. Esparza, S. Dubry, J. B. Benjamin, R. Volz, and P. Scuderi. 1989. Characteristics of tumor necrosis factor production in rheumatoid arthritis. Cell. Immunol. 122:131-145.

11. Morales-Ducret, J., E. Wayner, M. J. Elices, J. M. Alvaro-Gracia, N. J. Zvaifler, and G. S. Firestein. 1992. Alpha 4/beta 1 integrin (VLA-4) ligands in arthritis. Vascular cell adhesion molecule-1 expression in synovium and on fibroblast-like synoviocytes. J. Immunol. 149:1424-1431.

12. Johnson, B. A., G. K. Haines, L. A. Harlow, and A. E. Koch. 1993. Adhesion molecule expression in human synovial tissue. Arthritis Rheum. 36:137146.

13. Cronstein, B. N., and G. Weissmann. 1993. The adhesion molecules of inflammation. Arthritis Rheum. 36:147-157.

14. Molad, Y., J. Reibman, R. I. Levin, and B. N. Cronstein. 1993. A new mode of action for an old drug: colchicine decreases surface expression of adhe- sion molecules on both neutrophils (PMNs) and endothelium (EC). Arthritis Rheum. 35(Suppl. 9):S35a. (Abstr.)

15. Cronstein, B. N., S. C. Kimmel, R. I. Levin, F. Martiniuk, and G. Weissmann. 1992. A mechanism for the antiinflammatory effects of corticosteroids: the glucocorticoid receptor regulates leukocyte adhesion to endothelial cells and expression of endothelial-leukocyte adhesion molecule 1 and intercellular adhesion molecule 1. Proc. Natl. Acad. Sci. USA. 89:9991-9995.

16. Corkill, M. M., B. W. Kirkham, D. O. Haskard, C. Barbatis, T. Gibson, and G. S. Panayi. 1991. Gold treatment of rheumatoid arthritis decreases synovial expression of the endothelial leukocyte adhesion receptor ELAM-1. J. Rheumatol. 18:1453-1460.

17. Champion, G. D., G. G. Graham, and J. B. Ziegler. 1990. The gold complexes. Bailliere's Clin. Rheumatol. 4:491-534.

18. Harth, M. 1992. Mechanisms of action of disease modifying antirheumatic drugs. J. Rheumatol. (suppl. 32)19:100-103.

19. Harth, M., P. A. Keown, and J. F. Orange. 1983. Monocyte dependent excited oxygen radical generation in rheumatoid arthritis: inhibition by gold sodium thiomalate. J. Rheumatol. 10:701-707.

20. Scheinberg, M. A., L. M. Santos, and A. E. Finkelstein. 1982. The effect of auranofin and sodium aurothiomalate on peripheral blood monocytes. J. Rheumatol. 9:366-369.

21. Matsubara, T., and M. Ziff. 1987. Inhibition of human endothelial cell proliferation by gold compounds. J. Clin. Invest. 79:1440-1446.

22. Kawakami, A., K. Eguchi, K. Migita, H. Nakao, T. Otsubo, Y. Ueki, C. Shimomura, H. Tezuka, M. Matsunaga, T. Ishimaru, and S. Nagataki. 1990. Inhibitory effects of gold sodium thiomalate on the proliferation and interferongamma induced HLA-DR expression in human endothelial cells. J. Rheumatol. 17:430-435.

23. Jellum, E., E. Munthe, G. Guldal, and J. Aaseth. 1980. Fate of the gold and the thiomalate part after intramuscular administration of aurothiomalate to mice. Ann. Rheum. Dis. 39:155-158.

24. Rudge, S. R., D. Perrett, A. J. Swannell, and P. L. Drury. 1984. Circulating thiomalate after administration of disodium aurothiomalate: impurity or active metabolite? J. Rheumatol. 11:150-152.

25. Jellum, E., and E. Munthe. 1982. Fate of the thiomalate part after intramuscular administration of aurothiomalate in rheumatoid arthritis. Ann. Rheum. Dis. 41:431-432.

26. Windholz, M., S. Budavari, R. F. Bulmetti, and E. S. Otterbein. 1983. The Merck Index. Merck \& Co., Inc., Rahway, NJ. 1463 pp.

27. Jaffe, E. A. 1984. Culture and identification of large vessel endothelial cells. In Biology of Endothelial Cells. E. A. Jaffe, editor. Martinus Nijhoff, Boston. $1-13$.

28. Maciag, T., J. Cerundolo, S. Ilsley, P. R. Kelley, and R. Forand. 1979. An endothelial cell growth factor from bovine hypothalamus: identification and partial characterization. Proc. Natl. Acad. Sci. USA. 76:5674-5678.

29. Marks, D. C., L. Belov, M. W. Davey, R. A. Davey, and A. D. Kidman. 1992. The MTT cell viability assay for cytotoxicity testing in multidrug-resistant human leukemic cells. Leuk. Res. 16:1165-1173.

30. Chomczynski, P., and N. Sacchi. 1987. Single-step method of RNA isolation by acid guanidinium thiocyanate-phenol-chloroform extraction. Anal. Biochem. 162:156-159.

31. Sambrook, J., E. F. Fritsch, and T. Maniatis. 1989. Molecular Cloning: A Laboratory Manual. Cold Spring Harbor Laboratory, Cold Spring Harbor, NY.

32. Armatage, P., and G. Berry. 1987. Statistical Methods in Medical Research. Blackwell Scientific Publications, Oxford. 559 pp.

33. Carmer, S. G., and M. R. Swanson. 1973. An evaluation of ten pairwise multiple comparison procedures by Monte Carlo methods. Journal of the American Statistical Association. 68:66-74.

34. Aguilera, J. A., G. L. Newton, R. C. Fahey, and J. F. Ward. 1992. Thiol uptake by Chinese hamster V79 cells and aerobic radioprotection as a function of the net charge on the thiol. Radiat. Res. 130:194-204.

35. Munthe, E., and E. Jellum. 1983. Is gold necessary in so called chrysotherapy? Scand. J. Rheumatol. Suppl. 51:46-51.

36. Rudge, S. R., D. Perrett, and M. Kelly. 1988. Failure of oral thiomalate to act as an alternative to intramuscular gold in rheumatoid arthritis. Ann. Rheum. Dis. 47:224-227.

37. Chaudiere, J., E. C. Wilhelmsen, and A. L. Tappel. 1984. Mechanism of selenium-glutathione peroxidase and its inhibition by mercaptocarboxylic acids and other mercaptans. J. Biol. Chem. 259:1043-1050.

38. Jornot, L., and A. F. Junod. 1993. Variable glutathione levels and expression of antioxidant enzymes in human endothelial cells. Am. J. Physiol. 264:L482L489.

39. Pober, J. S., M. R. Slowik, L. G. Deluca, and A. J. Ritchie. 1993. Elevated cyclic AMP inhibits endothelial cell synthesis and expression of TNF-induced endothelial leukocyte adhesion molecule-1, and vascular cell adhesion molecule1, but not intercellular adhesion molecule-1. J. Immunol. 150:5114-5123.

40. Lazarevic, M. B., K. Yan, W. I. Swedler, M. M. Rasenick, and J. L. Skosey. 1992. Effect of gold compounds on the activity of adenylyl cyclase in human lymphocyte membranes. Arthritis Rheum. 35:857-864. 